\title{
The Natural History of the Sleep and Respiratory Engineering Track at EMBC 1988 to 2010
}

\author{
Ron S. Leder, Senior Member, IEEE, Gaston Schlotthauer, Member, IEEE, Thomas Penzel, Senior \\ Member, IEEE, Raimon Jané, Member, IEEE.
}

\begin{abstract}
Sleep science and respiratory engineering as medical subspecialties and research areas grew up side-by-side with biomedical engineering. The formation of EMBS in the 1950 's and the discovery of REM sleep in the 1950's led to parallel development and interaction of sleep and biomedical engineering in diagnostics and therapeutics.

Over the last 60 years, sleep investigators have relied on biomedical engineering to home-brew the equipment they used to measure physiology during sleep, because sensors and automatic sleep analysis and interpretation systems were too innovative to be available from traditional commercial sources.

The Sleep and Respiratory Engineering and related tracks of EMBC, between 1988 and 2010, have presented many papers on this topic including novel techniques of sleep interpretation and new equipment for diagnostics, portable and ambulatory measurement, monitoring, and therapeutics.

In this research, we summarize the activity at EMBC related to Sleep and breathing from an historical perspective, showing the number of publications and special sessions devoted to sleep and related topics.
\end{abstract}

\section{INTRODUCTION}

$\mathrm{D}$ URING the last seventeen years, and now, there is a direct funding mechanism for sleep research in the US. Thus, sleep science has a home of its own rather than being an orphan guest of various scientific disciplines. The National Center on Sleep Disorders Research (1993) at the NIH National Heart Lung and Blood Institute (NHLBI) is currently the central sleep research reference and funding mechanism. Since December 2000, there has been the National Institute of Biomedical Imaging and Bioengineering. Together with the National Center of Research Resources (NCRR), these agencies formalize cooperation in this field. Formerly, precedents of interdisciplinary cooperation between sleep science and biomedical engineering started much earlier; perhaps with the interaction of the electrical work of André Ampère and the physiology of Claude Bernard near Lyon France in the

Manuscript received April 23, 2010.

R. S. Leder. is with the Universidad Nacional Autononoma de Mexico, Mexico, DF 04510, Mexico (206984 2509; e-mail: rleder@ieee.org)

G. Schlotthauer is with the Universidad Nacional de Entre Ríos, and CONICET. (e-mail: gschlott@bioingenieria.edu.ar).

T. Penzel, is with Charité Center for Cardiology, Sleep Center, Charité University Hospital, Berlin, Germany (e-mail: thomas.penzel@charite.de)

R. Jané is with Institut de Bioenginyeria de Catalunya (IBEC), Dept. ESAII, Universitat Politècnica de Catalunya (UPC), and CIBER de Bioingeniería, Biomateriales y Nanomedicina (CIBER-BBN), Baldiri i Reixach 4, 08028, Barcelona, Spain (e-mail: raimon.jane@upc.edu).

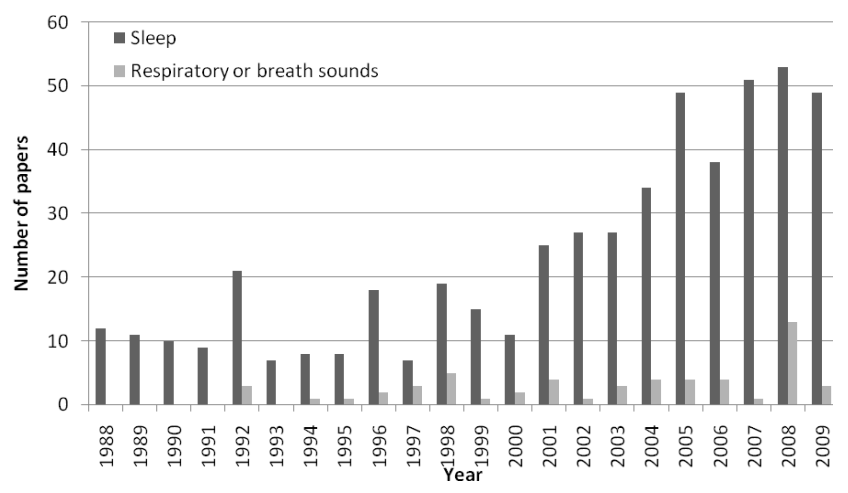

Fig. 1. 21 years of accepted papers at EMBC with the word sleep in (black) or breath sounds (grey) in the meta data of the articles. This is not the profile of only the sleep track but rather the profile of sleep at EMBC.

early $19^{\text {th }}$ century.

Even though Sleep Science is an established discipline, many clinical studies still do not consider the added variance of sleep and chronobiological factors in the data interpretation. Increasing knowledge about sleep and improving signal analysis techniques will increase sleep's impact on medical science.

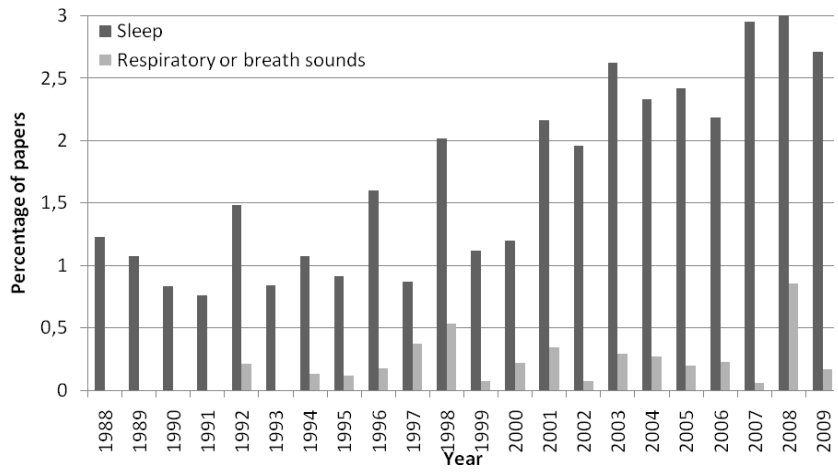

Fig. 2. 21 years of accepted papers at EMBC with the word sleep in (black) or breath sounds (grey) in the meta data of the articles. This is not the profile of only the sleep track but rather the profile of sleep at EMBC, expressed as \% of total papers; $1 \%$ to $3 \%$ over the years.

\section{DIAGNOSTIC EQUIPMENT}

Biomedical Engineering provided equipment for sleep studies like long term, minimally discomforting EEG 
electrodes, chest wall movement sensors, air flow sensors, static charge sensitive beds to noninvasively detect respiratory movement and ballistocardiogram, and respiratory sound measurement systems. Non invasive, unobtrusive methods for determining tidal volume during sleep were all essential for sleep studies. Estimation of expired volume via infrared imaging is probably one of the more creative biomedical engineering solutions, yet when the patient turns face down this method fails. One investigator (Stradling) video taped sleeping patients and then to automatically review body movements placed light sensors directly on the video screen to sense when and edge between the patient and the background moved; crude but effective.

Overnight, the transcutaneous oximeter and later the pulse oximeter became indispensible for sleep studies and for critical care medicine as well. The pulse oximeter was one of the first modern comprehensive medical instruments with long term data storage, graphic display, trend data presentation, and analog, digital, and wireless communication. The pulse oximeter is a tremendous biomedical engineering diagnostic equipment success story, in part driven by the needs of sleep disorders science.

Unobtrusive measures of physiology and vital signs continue to be improved by biomedical engineering for sleep studies. The Penaz principle was adapted by Ohmeda Inc. (Madison, WI) for measuring arterial blood pressure during sleep. The constant squeezing of a finger would keep the average person awake, yet a chronically sleep deprived patient with obstructive sleep apnea can tolerate the discomfort and sleep even though squeezing the same finger all night leads to discomfort. Biopac now has a twin Penaz cuff that alternates fingers to reduce discomfort. Many of these devices for sleep studies have been described in one of the sleep tracks at EMBC.

\section{AMBULATORY AND PORTABLE EQUIPMENT}

The (Norman) Holter, (M.D.) two channel ambulatory electrocardiogram was one of the earliest applications of ambulatory continuous long term physiological data collection. Sleep and chronobiology studies were the second applications using continuous, long-term physiological data collection. Today most systems are paperless, but 10 years ago an overnight sleep study used 1000 pages of 16 inch to 22 inch wide z-folded paper. Earlier attempts at a more modern, multichannel, long term continuous physiological recording system related to sleep disorders included:

The Vitalog (Laughton Miles; CA, US) the MESAM (Thomas Penzel, Marburg, DE), Flaga Embla (Reykjavick, IS, now CO, US), Edentrace (AU, MN US), Honeywell (MN, US), Cadwell Systems (WA, US), Biologic (IL, US), and more.

There is an understandable trend toward noninvasive sleep measurement systems and biomedical engineering has provided such systems. For automatic classification of the severity of obstructive sleep apnea syndrome (OSAS),

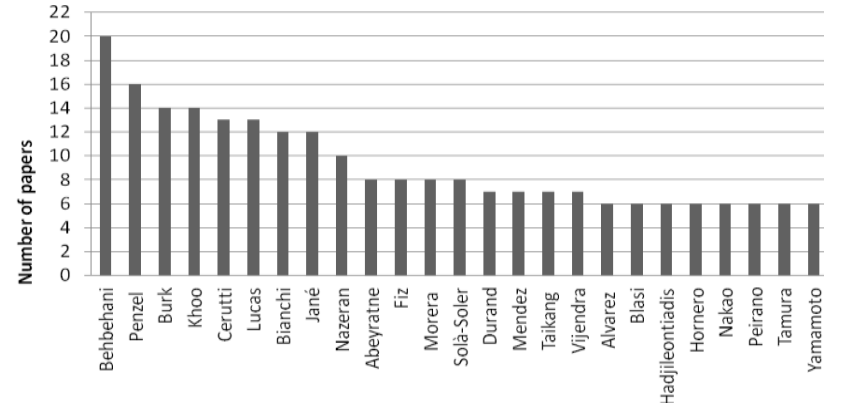

Fig. 3. Over the 21 years since 1988 some few authors have submitted the majority of sleep papers. Here are the authors who have submitted and published 6 or more sleep papers. We should also mention that there is a group of authors who have submitted the majority of papers on the theme of analyzing breath sounds.

snoring measurement systems have been developed. One such recent device is the Snoryzer Uno (Sibel SA, Barcelona, Spain). This approach uses continous measurement and analysis of snore sound intensity and several snore frequency parameters to classify the severity of OSAS. Now that obstructive sleep apnea is known to have a clinically significant prevalence in the general population, sophisticated yet simple to use devices, are important for screening studies and for patient follow up.

\section{THERAPEUTICS}

On the treatment side, obstructive sleep apnea syndrome is one of the sleep disorders for which there is a direct and mechanical treatment; a pneumatic splint for the upper airway provided by nasal continuous positive airway pressure (nCPAP). Since the first primitive nasal CPAP machine introduced by Colin Sullivan in 1979, Biomedical Engineers have refined the technology to provide a more comfortable experience for the patients which in turn improves compliance. Obviously, the sleep track is not the only place in which Biomedical Engineering has made the difference in clinical practice and research. In fact Biomedical Engineering is present almost everywhere in health sciences.

\section{HISTORICAL PERSPECTIVE}

The underpinnings of the discovery of REM sleep in the 1950 's were laid in the thirties with the vacuum tube powered differential amplifier for measuring EEG, such as those manufactured by Albert and Ellen Grass of the Grass Instruments Co Quincy, MA, now part of AstroMed (Warick RI.). This early contribution of EEG signal acquisition together with the FM tape recorder opened modern research on computer analysis of EEG including sleep. The FM tape recorder allowed slow replay of EEG data so that slow computers could keep up processing the time series.

Recorded history for EMBC via IEEE Xplore starts in 1988. Since then, one can see steady growth in the number or 
articles with the word sleep in the meta data accepted by EMBC. Eleven years earlier, in 1977, there were already 5 major sleep centers in the US.

Figures 1 and 2 are not a true profile of the Sleep Disorders and Respiratory Engineering track, but of the number of articles related to sleep via the word "sleep" and phrase "breath sounds", respectively, in the meta data of the articles.

There are other EMBC tracks that have sleep related articles and sleep appears in three or four contexts in the approximately 550 semi-controlled key words of the currently unfolding EMBC 2010 meeting.

In 1990 and 1991 there was an EMBC track entitled "Sleep and Respiratory Control Dynamics". In 1992, track 54 was "Sleep, EEG and Related Disorders". In the following years, there was not a specific track for sleep \& respiratory eng., until 2003 (in Cancun). Then, there was a track on "Current Issues in Sleep Apnea". Since then, the track was present with several names:

2003: "Current Issues in Sleep Apnea"

2004: "Respiratory Systems and Sleep Apnea"

2005: "Theme 15: Respiratory System Engineering and SARS" Chair(s): M. Khoo, G.P. Zhao, C.S. Poon, J.Hu"

Tracks: Sleep Apnea I: Novel Methods of Detection and Assessment

Sleep Apnea II: Cardiovascular Variability

Modeling the Respiratory Control System

Bioengineering Methods in SARS

Respiratory Systems Engineering

2006: 9.3.1 Sleep Apnea (chair: Thomas Penzel)

2007: Sleep Disorders I Oral Session

Chair: Behbehani, Khosrow

Sleep Disorders II (poster session)

2008: Sleep Apnea Chair: Behbehani, Khosrow, Univ. of

Texas at Arlington

Co-Chair: Raimon Jané

2009: Sleep disorders and respiratory engineering (CSAS, OSAS etc) Chair: Thomas Penzel

2010: Sleep disorders and respiratory engineering (CSAS, OSAS etc) and Invited session on sleep disorders and respiratory engineering. Co Chairs: Ron Leder and Gaston Schlotthauer.

Notably, 1999 was the first EMBS/BMES joint meeting, 2000 was a joint meeting with the World Congress on Medical Physics, and 2002 was the second joint EMBS/BMES meeting. At this meeting there was an invited special session on sleep and breathing organized by Michael Khoo and John Clark, with presentations by John Remmers, Ron Harper, John Orem, and Michael Khoo.

Between 1991 and 2003 EMBC did not consider sleep as having a home of its own and it was not until the conference Computers in Cardiology was having an annual apnea detection challenge via heart rate variability that sleep seemed more important in its own rite at EMBC.
IEEE Computers in Cardiology is an annual meeting that has been held for many years. In 2000 the meeting was held in Boston and was organized by Roger Marks and George Moody. Ary Goldbereger promoted the idea of a competition on computer methods applied to cardiology problems. Sleep apnea detection via a single ECG lead was the first challenge and because of its popularity has continued to be an annual event. This initiative was supported by Physionet sponsored by the US National Institutes of Health. Data were from 70 patient nights. 35 nights were training data and 35 were test data all recorded and scored in Dr. Thomas Penzel's sleep laboratory in Marburg Germany. Data consisted of normal, moderate, and severe sleep apnea. The first task was to identify all subjects with severe sleep apnea and the second task was to detect and classify the minutes with and without disordered breathing. Twelve groups participated and four did identify all subjects correctly. The winners had $92 \%$ correct classifications of minutes. This competition started a lot of research to detect sleep apnea from the ECG using electrocardiographic derived respiration. Sleep apnea is now a regular topic at the IEEE Computers in Cardiology conference.

Figure 3, presents the authors that have submitted the majority of sleep papers, over the 21 years since 1988. Only included are authors who have submitted and published 6 or more sleep papers. The grouping of authors is not shown. We mention that there is one group of authors who have submitted the majority of papers at EMBC on the theme of analyzing breath sounds.

Browsing the approximately 550 keywords in this year's EMBC list, one observes the key word sleep and related keywords in Cardio and pulmonary regulation, Neurological disorders, Human performance, and Therapeutics and diagnostic systems. We hope sleep science continues to advance such that it maintains a home of it's own at EMBC.

\section{CONCLUSION}

Since sleep and respiratory clinicians and basic scientists interact with Biomedical Engineers in day to day activities, it could be important to promote interdisciplinary cooperation in a professional and scientific forum like EMBC. There are certain aspects of the EMBC meeting model that make it a challenge for special session chairs to get the right mix of people for the session theme. With a view toward EMBC 2011 in Boston, where there are many sleep professionals, and the observation that Sleep is covered from different perspectives in the world wide sleep societies, (APSS, SRS, APT, WSF), the physiologists (APS FASEB) the physicians (ACCP, ATS) and international counterparts, we see the mutual benefit in bringing these perspectives together with Biomedical Engineering at EMBC. We hope that there can be a poster symposium format in addition to an oral symposium that optimizes the interaction of these different perspectives on the familiar stranger, sleep, which takes up about $30 \%$ of our lives. 


\section{REFERENCES}

[1] IEEE Xplore database. 1988 to2010.

[2] T. Penzel, Personal communication April-2010.

[3] G. Schlotthauer, Personal communication April 2010.

[4] R. Leder, Personal communication April 2010.

[5] R. Jané, Personal communication June 2010.

[6] <http://www.nhlbi.nih.gov/about/ncsdr/index.htm>

[7] <http://www.wfsrsms.org/> 